GRASSLAND ECOLOGY

Rare plants and rainfall

Ecol. Lett. 18, 1293-1300 (2015)

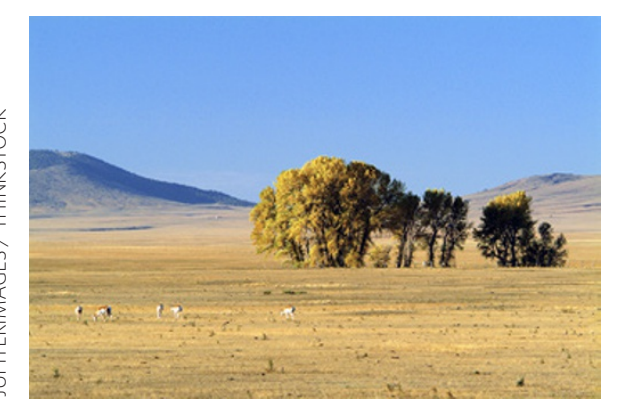

Year-to-year variations in rainfall are projected to increase as the climate warms, with uncertain consequences for ecosystem structure and function. Results from a rainfall manipulation experiment suggest that more variable rainfall regimes will lead to more diverse grassland communities, which can buffer concomitant declines in dominant plant productivity.

In a six-year rainfall manipulation experiment, Laureano Gherardi and Osvaldo Sala, of Arizona State University, assessed the effect of interannual variations in rainfall on plant functional diversity and productivity in a desert grassland in New Mexico. Plant functional diversity increased with rainfall variance, underpinned by a reduction in the relative abundance of perennial grasses (the dominant plant functional type) and an increase in the abundance of rarer plant functional types, such as shrubs, annual grasses and forbs. The contrasting responses of dominant and rare species seemed related to the fact that dominant species did better in terms of productivity under modal rainfall regimes, whereas rare species did better under very wet conditions.

Using structural equation modelling, the researchers show that the rise in the abundance and productivity of rare plants as rainfall regimes grew more variable partially offset the concomitant drop in perennial plant productivity.

\section{SYNTHETIC BIOLOGY}

\section{Elegans control by auxin}

Development http://doi.org/9c8 (2015)

A fundamental strategy used to study the function of proteins in a complex biological system is to make them 'appear' and 'disappear' at will, and then analyse the consequences. Conditional expression in eukaryotes is relatively easy, but controlled and targeted depletion is difficult to achieve. To this end, Abby Dernburg at the Lawrence Berkeley National Laboratory and colleagues transferred the plant auxin perception system into living nematodes.

Auxin receptors such as TIR1 connect auxin perception with the protein ubiquitination pathway. In the presence of auxin, TIR1 recognizes a degradation sequence (degron) in Aux/IAA transcriptional regulators. Non-plant eukaryotes do not have an auxin receptor, but do contain the ubiquitination machinery. Introducing a high-affinity version of Arabidopsis thaliana TIR1 and a small degron from
SELF-INCOMPATIBILITY Avoiding inbreeding in Arabidopsis Science 350, 684-687 (2015)

Plants have evolved many ways to avoid self-fertilization - including a number of different self-incompatibility systems, which recognize genetically similar pollen and prevent its growth. Now Zongcheng Lin and colleagues working at the University of Birmingham, UK, have demonstrated that the self-incompatibility mechanism from one group of plants can be functional in another.

The self-incompatibility system of field poppies (Papaver rhoeas) has two main components - a pollen protein, PrpS, and a signal protein, PrsS, produced by the stigma encoded by closely linked genes within the Slocus. When PrpS recognizes PrsS of its own type, it triggers a cell death program within the pollen to abort pollination. But if PrsS does not match, 'self' recognition does not occur and pollination can proceed. Lin and colleagues engineered the normally self-fertile Arabidopsis thaliana to express the two poppy genes in pollen and pistils, respectively, creating plants that rejected their own pollen while allowing fertilization by pollen that either does not express PrpS or expresses PrpS of a different class.

It is remarkable that so few genes are needed to implement the poppy selfincompatibility system in Arabidopsis, as these two plants diverged from a common ancestor around 140 million years ago. Similar engineering might be a route to ensure outcrossing in crop breeding.
IAA17 into Caenorhabditis elegans caused auxin-dependent degradation of several worm proteins.

The effect is fast, specific and, importantly, reversible. The strategy works during various developmental stages, for both cytoplasmic and nuclear proteins, and can be made tissue-specific. Auxin, as an inducer, is a small non-toxic molecule that easily diffuses through membranes. The system results in more penetrant phenotypes than RNAi and allows precise timing of degradation, all with minimal side effects. It is likely that we may soon see auxin vials in worm labs.

\section{FLOWER DEVELOPMENT}

\section{Sex lessons from cucumbers} Science 350, 688-691 (2015)

Most angiosperms produce bisexual flowers, but some species have male and female flowers, either on the same or separate individuals. A newly identified gene responsible for determining the sex of cucumber and melon flowers may show how unisexual flowers and dioecious species develop.

Abdelhafid Bendahmane of the Institut National de la Recherche Agronomique, France, and colleagues identified a gene, ACS11, whose loss in cucumbers and melons resulted in the production of only male flowers. ACS1 1 encodes a rate-limiting enzyme for ethylene biosynthesis, and the mutant phenotype could be rescued by exogenously supplying ethylene. ACS11 is specifically expressed in the buds and vascular bundles of female flowers, suggesting that ACS11-mediated ethylene production is needed for female flower development.

$A C S 11$ also represses the carpel inhibitor, $W I P 1$, which in turn represses the stamen inhibitor, ACS7. The lack of ACS11 results in exclusively male flowers by allowing WIP1 to directly prevent carpel formation and indirectly stimulate stamens. Expression of ACS11 releases the block on carpel development while restoring the suppression of stamens, thereby leading to female flowers. Disrupting the function of ACS7 allows both carpels and stamens to develop, producing hermaphroditic flowers.

The researchers further created a dioecious mating system by crossing two individuals with different allelic combinations of ACS11 and WIP1, demonstrating a possible path for dioecy to evolve from monoecy.

Written by Anna Armstrong, Jun Lyu, Chris Surridge and Guillaume Tena. 